\title{
Further defining the language impairment of autism: Is there a specific language impairment subtype?
}

\author{
Andrew J.O. Whitehouse*, Johanna G. Barry, Dorothy V.M. Bishop \\ Department of Experimental Psychology, Oxford Study of Children's Communication Impairments, \\ University of Oxford, South Parks Road, Oxford OX1 3UD, United Kingdom
}

Received 1 May 2007; received in revised form 19 November 2007; accepted 14 January 2008

\begin{abstract}
Some children with autism demonstrate poor nonword repetition-a deficit considered to be a psycholinguistic marker of specific language impairment (SLI). The present study examined whether there is an SLI subtype among children with autism. We compared the language abilities of children with SLI $(n=34, M$ age $=11 ; 10$ S.D. $=2 ; 3)$, and children with autism with (Apoor, $n=18, M$ age $=10 ; 11$ S.D. $=3 ; 1$ ) and without (Aapp, $n=16, M$ age $=10 ; 8$ S.D. $=2 ; 7$ ) structural language difficulties. Participants were administered battery of standardized language and memory tests. Although there were some similarities in the language profile of the SLI and Apoor groups, the two groups differed on the tests of oromotor ability and verbal short-term memory and showed a different pattern of errors on the nonword repetition task. These findings providing evidence against the idea of an SLI subtype in autism. Further analyses suggested that the nonword repetition deficits experienced by some children with autism may arise when there is substantial impairment in multiple autistic domains.
\end{abstract}

Learning outcomes: Readers will be introduced to (a) the current state of behavioral, cognitive and genetic research that has investigated the relation between SLI and autism, and (b) three hypotheses of why there exists similarity in the language characteristics of children with SLI and autism. Readers will then be taken through a detailed comparison of the language and memory abilities of group of children with each diagnosis. A theoretical model that seeks to explain the relation between these two disorders will be discussed.

(C) 2008 Elsevier Inc. All rights reserved.

* Corresponding author. Tel.: +44 1865271 334; fax: +44 1865281255.

E-mail address: andrew.whitehouse@psy.ox.ac.uk (A.J.O. Whitehouse). 
Autism is a pervasive developmental disorder characterized by deficits in social behavior, communication and by restricted and repetitive behavior and interests (American Psychiatric Association, 1994). Although an individual must show impairment in all three autistic 'domains' to receive a diagnosis of autism, there is considerable phenotypic heterogeneity in this population. This variability has led to the claim that the diagnostic classification of autism incorporates a number of subtypes (Fein et al., 1999; TagerFlusberg \& Joseph, 2003).

Communication deficits are perhaps the most frequently observed deficit in individuals with autism. Pragmatic deficits such as repetitive use of language and limitations in ability to initiate and sustain conversations are pervasive in this population. However, there is large variability in structural linguistic skills (i.e., phonology, grammar, vocabulary). Although many individuals with autism display severe deficits with expressive and receptive language, a significant minority of individuals appear to be without any linguistic abnormality. Recent evidence suggests that this variability may be explained by the existence of a subtype of individuals with autism who have core impairment in linguistic ability similar to that seen in specific language impairment (SLI) (Tager-Flusberg, 2006; Tager-Flusberg \& Joseph, 2003).

Kjelgaard and Tager-Flusberg (2001) administered a range of language tests to a large group of children with autism aged between four and 13 years. Participants were divided into three groups based upon their performance on the Clinical Evaluation of Language Fundamentals-III (Semel, Wiig, \& Secord, 1995): (1) normal language, (2) borderline language and (3) language-impaired. The language-impaired subgroup was characterized by broad deficits in syntactic and semantic ability; a language profile similar to that observed in children with SLI. However, the most striking finding was the poor performance by both the borderline and language-impaired groups on a task of nonword repetition, which is a deficit thought to be a defining feature of the SLI phenotype (TagerFlusberg \& Cooper, 1999).

SLI is recognized when a child fails to develop language according to the normal time frame despite adequate hearing, intelligence and physical development. Although there is considerable heterogeneity of symptoms in SLI, difficulties are often seen in both the expressive (e.g., syntactic, vocabulary, phonological and/or oromotor deficits) and receptive (comprehension deficits) domains (Bishop, 1997).

There are numerous theoretical accounts of SLI (for a review, see Bishop, 1997). One of the most prominent explanations is that the selective language impairment is the result of deficits in phonological short-term memory (STM) (Gathercole \& Baddeley, 1990). Deficits in phonological STM are thought to delay language learning by limiting one's ability to learn novel words and comprehend new syntactic structures (Gathercole \& Baddeley, 1990). Phonological STM is typically gauged through tasks of immediate repetition of verbal information. Tasks in which participants are asked to repeat nonwords (as used by Kjelgaard \& Tager-Flusberg, 2001) are particularly sensitive to deficits, as individuals cannot draw on previous experience to help them complete the task. Children with SLI are typically able to repeat short nonwords accurately; however, their performance decays with nonwords of three or more syllables, suggesting that the difficulty is in holding novel phonological material in memory, rather than in basic aspects of perception and production. Previous studies have suggested that around three-quarters of 
children with SLI have considerable difficulties with nonword repetition (Conti-Ramsden, Botting, \& Faragher, 2001; also see meta-analysis by Graf-Estes, Evans, \& Else-Quest, 2007).

Nonword repetition has gained particular importance in SLI research not only because deficits are seen in many children with current evidence of language difficulties, but also because poor performance is observed in older children whose language impairment has resolved (Bishop, North, \& Donlan, 1996). This evidence has led a number of researchers to suggest that poor nonword repetition may act as a psycholinguistic marker of the SLI phenotype (Bishop et al., 1996; Conti-Ramsden et al., 2001; Tager-Flusberg \& Cooper, 1999). For this reason, the finding that language-impaired children with autism also show poor nonword repetition (Kjelgaard \& Tager-Flusberg, 2001), has raised suggestions that there is subtype of children with autism who show the same neurocognitive phenotype as that seen in children with SLI (Tager-Flusberg \& Joseph, 2003).

The notion of an SLI subtype in autism has stimulated interest as to whether there is etiological overlap between SLI and autism. So far, this proposal has received mixed evidence. Tomblin, Hafeman, and O'Brein (2003) found that the prevalence of autism in siblings of children with SLI (1\%) was significantly higher than general population estimates. However, the sample size was very small for an epidemiological study, and the rate of autism cases in siblings of children with SLI did not significantly differ from that of the control group $(0.4 \%)$. Molecular genetic studies investigated whether there was overlap in the loci associated with the SLI and autism phenotypes. Evidence emerged that autism may be associated with a mutation of the FOXP2 gene on chromosome 7q (Alarcón et al., 2002), which is known to co-segregate with a specific form of speech and language impairment in one British family (Lai, Fisher, Hurst, Vargha-Khadem, \& Monaco, 2001). However, these findings have failed to be replicated (Wassink et al., 2002), leading researchers to conclude that FOXP2 is unlikely to be implicated in autism (Newbury et al., 2002). A number of family studies have found elevated levels of self-reported language difficulties in relatives of individuals with autism (e.g., Folstein et al., 1999). However, these findings have not been replicated when relatives are tested with standardized language assessments (Bishop et al., 2004; Folstein et al., 1999; Pilowsky, Yirmiya, Shalev, \& Gross-Tsur, 2003; Whitehouse, Barry, \& Bishop, 2007). In one of these studies, our research group (Whitehouse et al., 2007) examined the linguistic and pragmatic abilities of parents of children with autism and parents of children with SLI. The two parent groups showed a double dissociation between structural language skills (intact in parents of children with autism and impaired in parents of children with SLI) and social communication ability (intact in parents of children with SLI and impaired in parents of children with autism), indicating that, while structural language impairments are part of the heritable communication deficit in SLI, this is not the case with autism.

The lack of conclusive evidence regarding etiological overlap between autism and SLI, has led researchers to consider alterative positions for why some children with autism have difficulty with nonword repetition. One possibility is that poor nonword repetition ability in some individuals with autism is due to the output demands of the task. Although the nonword repetition task is highly effective as a quick test for identifying weak phonological STM in SLI, its apparent simplicity is deceptive. Poor performance can arise for different reasons. For instance, it may be that children with autism do poorly because of 
difficulty in programming the articulatory apparatus to execute a long and complex sequence of movements. Some evidence for this position comes from Page and Boucher (1998), who found a high incidence of oromotor impairments in a sample of 33 individuals attending a special school for children with autism.

A further possibility for why some children with autism have nonword repetition difficulties was outlined in Whitehouse et al. (2007). In this study we proposed that there was no etiological overlap between SLI and autism; whereas nonword repetition deficits in SLI result from genetic factors affecting neuroanatomical pathways responsible for language, nonword repetition deficits in the autistic population are associated with broader autistic symptoms. In particular, we suggested that structural language deficits - including poor nonword repetition - may arise when there is a particular conjunction of behavioral/ cognitive deficits, each of which would not disrupt nonword repetition on its own, but when they occur in combination would lead to impaired ability to do the task. For instance, poor attention to others' speech coupled with difficulty imitating could impair nonword repetition, but for quite different reasons than those seen in SLI. DSM-IV guidelines state that every individual with an autism diagnosis has deficit in all three autistic domains (American Psychiatric Association, 1994). However, behavioral genetic studies have provided some evidence that these deficits can be fractionated into the three component parts, each of which operates independent of the others (Ronald et al., 2006; but see Constantino \& Todd, 2003); an individual can be impaired to a greater or lesser extent on the three separable components. In Whitehouse et al. (2007), we suggested that it is not the mere presence of deficit in multiple autism domains that make individuals more susceptible to nonword repetition deficits, but rather the presence of substantial deficit in multiple domains.

We set out to test three competing hypotheses for why some children with autism have nonword repetition deficits. The first hypothesis is that nonword repetition deficits in autism reflect etiological overlap with SLI (Kjelgaard \& Tager-Flusberg, 2001). The second position is that nonword repetition deficits in autism are due to some children having difficulties programming speech-motor movements, rather than poor phonological STM. The third position is that nonword repetition deficits are associated with greater severity of autistic symptomatology (Whitehouse et al., 2007).

The current study sought to test between these various positions by comparing the language profiles, oromotor skills, and autism-related behaviors of subgroups of children with autism and SLI. To test the first hypothesis, we compared the language profile of children with SLI, with the language profile of two subgroups of children with autism, selected based upon the presence or absence of structural language impairment. Although Kjelgaard and Tager-Flusberg showed that language impaired children with autism showed the same broad syntactic and semantic deficits that are observed in children with SLI, they provided little information on other areas of language functioning. The current study examined abilities across the entire language profile, spanning structural language abilities, verbal short-term memory and pragmatic language skills. The first hypothesis predicts that children with autism who also have structural language impairment will have a similar language profile to children with SLI. Furthermore, if poor nonword repetition in languageimpaired children with autism is caused by a deficit in phonological STM (as is the case for children with SLI), then we would expect these two groups to show a similar pattern of 
errors on the task of nonword repetition. To test for the second and third hypotheses, we divided the children with autism according to their performance on the test of nonword repetition. The second hypothesis predicts that the children with autism who have poor nonword repetition will perform worse than the remaining children with autism on tasks demanding complex articulatory planning (i.e., the task of oromotor ability). The third hypothesis predicts that there will be quantitative differences in autistic symptomatology between those children with and without nonword repetition deficits.

\section{Method}

\subsection{Participants}

In total, 68 children participated in this study: 34 children with SLI (10 female and 24 male) and 34 children with autism (1 female and 33 males).

Children in the SLI group were aged between 6 and 15 years and were recruited from special schools for children with language impairment or support units in mainstream schools. Children were included in this group if they (1) performed below the 10th percentile on at least two of the following standardized tests: Test for Reception of GrammarElectronic (Bishop, 2005), Expression, Reception and Recall of Narrative Instrument (Bishop, 2004), the sight word and phonemic decoding subtests of the Test of Word Reading Efficiency (Torgensen, Wagner, \& Rashotte, 1999), the Global Communication Composite of the Children's Communication Checklist-2 (Bishop, 2003), repetition of nonsense words subtest of the NEPSY (Korkman, Kirk, \& Kemp, 1998), memory for sentences subtest of the NEPSY (Korkman et al., 1998); (2) had a nonverbal IQ of 80 or above, as measured by the Wechsler Abbreviated Scale of Intelligence (WASI; Wechsler \& Chen, 1999); (3) did not have a diagnosis of any developmental disorder other than SLI; and (4) they were able to hear a pure tone of $20 \mathrm{~dB}$ or less in each ear, at 500, 1000, 2000 and $4000 \mathrm{~Hz}$. The Social Communication Questionnaire (Rutter, Bailey, \& Lord, 2003) was used to exclude autism in any children where there was diagnostic uncertainty. Further information on this checklist is provided below.

The children with autism were aged between 7 and 15 years. Each child had a DSM-IV based diagnosis of autism (American Psychiatric Association, 1994), which was confirmed with the Autism Diagnostic Observation Schedule-Generic (ADOS-G; Lord et al., 2000). All children met ADOS-G criteria for autism except for two, who met criteria for an autism spectrum disorder. Participants were included in this study only if their nonverbal IQ (NVIQ) was higher than 80, as measured by the WASI, and they passed the hearing test described above.

The children with autism were divided into two subgroups based upon the same criteria used to define language impairment in the SLI group (i.e., had a score below the 10th centile on two or more of the standardized language tests listed above). There were 18 children with autism who were deemed to have language impairment (Apoor), and 16 children with autism who had appropriate language skills (Aapp).

Separate one-way ANOVAs were conducted to test for between-group differences in the chronological age and nonverbal IQ of the SLI, Apoor and Aapp groups (see Table 1). 
There was no significant difference between the three groups for chronological age, $F(2,65)=1.44, p=.24$. There was, however, a significant difference in nonverbal IQ, $F(2,65)=3.5, p<.05$. Scheffé post hoc tests revealed a trend for the Aapp group to have a higher NVIQ than the SLI $(p=.05)$ and Apoor groups $(p=.09)$. There was no difference in NVIQ between the SLI and Apoor groups $(p=1)$.

\subsection{Procedure}

This research was approved by the Central University Research Ethics Committee of Oxford University. All participants and their parents were fully informed about the study procedure and were aware of their right to withdraw at any time.

Participants completed a battery of standardized language tests that assessed verbal STM, receptive and expressive language, oromotor ability and social communication ability. Testing took place in a quiet room either at Oxford University or at the participants' home/school.

\subsection{Assessments}

Receptive language was assessed with a computerized version of the Test for Reception of Grammar-Electronic (TROG-E, Bishop, 2005). Participants were shown four pictures and simultaneously heard a phrase or sentence. They were required to select the picture that best represented the sentences they had heard.

Expressive language was measured with the Beach Story from the Expression, Reception and Recall of Narrative Instrument (ERRNI, Bishop, 2004). Participants were asked to familiarize themselves with a series of 15 pictures that told a narrative. Following this, the participants were required to tell a story based on the pictures. After a 15-30 min interval, participants re-told the story, this time without the aid of the pictures. The assessment provided a measure of grammatical complexity (MLUw): the mean number of words in each utterance, averaged over the two narrative tellings.

The oromotor sequences task from the NEPSY test battery (Korkman et al., 1998) provided a measure of speech motor ability. This task requires participants to repeat phrases that are difficult to articulate (e.g., 'squish squash') five times. Six of the 14 items involve the repetition of short tongue twisters (e.g. 'put the pepper beads in the paper bag') and may also tax STM skills (Barry, Yasin, \& Bishop, 2007).

Phonological STM was assessed using the repetition of nonsense words subtest from the NEPSY. Thirteen nonsense words ( 3 two-syllable nonwords, 3 three-syllable nonwords, 4 four-syllable nonwords and 3 five-syllable nonwords) were pre-recorded by a female with a British accent. Participants heard the words through headphones and were required to immediately repeat each item. Participants were awarded 1 point for each syllable repeated correctly. A standard score of 8 or less was adopted as the criterion for poor performance on this test. Although this is less than one standard deviation from the mean (of 10), norms for the NEPSY were derived from US standardization. At present there are no standard scores based on UK children. The current threshold was chosen as it was sufficiently below the mean of a sample of typically developing children recruited previously by our research group ( $n=33, M=11.6$, S.D. $=2.2$, see Barry et al., 2007). 
Verbal STM was measured with the memory for sentences subtest from the NEPSY. For this task, participants were orally presented with a list of sentences of increasing length and grammatical complexity. Participants were required to immediately repeat each sentence. Scores were based upon the frequency of recall errors (two points for a correctly repeated sentence; one point for a repetition containing no more than two errors; otherwise, no points).

Parents of each participant completed the Children's Communication Checklist-2 (CCC-2; Bishop, 2003), a 70-item questionnaire designed to assess both the structural and pragmatic aspects of a child's communicative ability. Four subscales measure structural language skills [i.e., (A) speech, (B) syntax, (C) semantics, (D) coherence], four measure pragmatic language skills [i.e., (E) inappropriate initiation, $(\mathrm{F})$ stereotyped language, $(\mathrm{G})$ use of context, $(\mathrm{H})$ nonverbal communication] and two measures other autistic-like behavior [i.e., (I) social relations, (J) interests]. Two summary measures are computed. A general communication composite (GCC) is based on a summed score of the eight subscales relating to communication $(\mathrm{A}-\mathrm{H})$. A high score on this measure indicates greater communicative competence. The social-interaction deviance composite (SIDC) provides an index of communicative profile: it reflects the relative extent of social communication difficulties versus structural language deficits. This measure is computed by subtracting the sum of the two pragmatic and two social interaction subscales (i.e., $E+H+I+J$ ) from the subscale scores that taps structural language difficulties (i.e., $A+B+C+D$ ). A positive score is indicative of a child with predominantly structural language difficulties, while a negative score would denote a child with pragmatic/social difficulties that are disproportionate to structural language impairments.

Finally, parents of the children with autism also completed the Social Communication Questionnaire (SCQ; Rutter et al., 2003). The SCQ is a 40-item questionnaire based on the Autism Diagnostic Interview-Revised (Lord, Rutter, \& LeCouteur, 1994) and obtains information about core autistic symptomatology. Each item asks about the child in question and the informant must check either 'yes' or 'no' as to whether that behavior is/was demonstrated by the child. The response is then assigned a point rating of 1 (presence of abnormal behavior) or 0 (absence of abnormal behavior). The SCQ can be divided into three subscales relating to the three core autistic domains: (1) reciprocal social interaction; (2) communication; (3) restricted, repetitive and stereotyped patterns of behaviors. Thirtysix of the 40 questions are categorized into these three subscales.

\section{Results}

Standard scores were computed for participants' performance on each task. Standard scores on the MLUw (ERRNI) and the TROG-E were based around a mean of 100 and a standard deviation of 15, while standard scores for the NEPSY tasks and the CCC-2 subscales were based around a mean of 10 and a standard deviation of 3 . Prior to comparing data between groups, univariate normality (Kolmogorov-Smirnov test of normality, $p<.05)$ was tested for the four groups' performance on each behavioral task, as well as the GCC and SIDC of the CCC-2. Participants' performances on the TROG-E were nonnormally distributed. This data distribution was normalized via logarithmic transformation (base 10). 
Table 1

Details of the SLI participants as well as those participants with autism with (Apoor) and without language impairment (Aapp)

\begin{tabular}{llll}
\hline & SLI $(n=34)$ & Apoor $(n=18)$ & Aapp $(n=16)$ \\
\hline Sex & 24 male, 10 female & 17 male, 1 female & All male \\
Chronological age & $11 ; 10(2 ; 3)$ & $10 ; 11(3 ; 1)$ & $10 ; 8(2 ; 7)$ \\
Range & $6 ; 7-15 ; 8$ & $7 ; 2-15 ; 10$ & $7 ; 6-15 ; 10$ \\
Nonverbal IQ & $100.35(13.21)$ & $100.33(11.74)$ & $110.34(14.88)$ \\
Range & $80-137$ & $80-123$ & $93-137$ \\
\hline
\end{tabular}

Means and ranges are presented for both chronological age and Nonverbal IQ, with S.D. within parentheses.

Hypothesis 1. An SLI subtype among children with autism?

The first hypothesis predicts that the SLI and Apoor groups will (a) have similar linguistic profiles and (b) show a similar pattern of errors for the task of nonword repetition. Between-group differences were assessed in three stages: first for the tasks of verbal and phonological STM, receptive language, expressive language and oromotor ability, followed by the various measures from the $\mathrm{CCC}-2$, and finally the pattern of errors made on the nonword repetition task.

\subsection{Structural language and STM tasks}

The three groups' performance on the nonword and sentence repetition tasks, the TROG-E, the MLUw (ERRNI) and the oromotor sequences task were analyzed with MANOVA and Scheffé post hoc tests (see Table 2). A significant main effect for group emerged from the analysis, $F(10,122)=6.59, p<.001$, Wilks' $\lambda=.42$, partial eta ${ }^{2}=0.35$. While the Aapp group had significantly better performance than the SLI group on the test of nonword repetition, there was no difference between the SLI and Apoor groups. There was also no significant difference between the SLI and Apoor groups on the MLUw and TROG-E tasks. The Apoor group had significantly better performance than the SLI and Aapp groups on the oromotor sequences task, while both autism subgroups performed better than the SLI group on the task of sentence repetition.

\subsection{Children's communication checklist—second edition}

CCC-2 questionnaires were not completed for three participants in the Aapp group and one participant in the Apoor group. MANOVA investigated scores on each CCC-2 subscale for the remaining participants (see Fig. 1). The analysis revealed a main effect for group, $F(20,104)=5.3, p<.001$, Wilks' $\lambda=.25$, partial eta $^{2}=0.51$. There were no group differences on the subscales measuring speech, syntax and semantics (for each subscale, $p>.1$ ). There were significant group differences for all other subscales (coherence subscale: $p<.05$; remaining subscales: $p<.001$ ). For the coherence and stereotyped language subscales, the Aapp group (but not the Apoor group) had a lower standard score than the SLI group (for both comparisons, $p<.01$ ). The two autism groups had significantly lower standard scores than the SLI group on the remaining subscales (all $p \mathrm{~s}<.01$ ). 
Table 2

Means and S.D. (within parentheses) for the performance of the SLI, Apoor and Aapp groups on the battery of memory and language assessments

\begin{tabular}{|c|c|c|c|c|c|c|c|}
\hline Domain & Assessment & SLI & Apoor & Aapp & Scheffé post hoc analyses & Partial eta ${ }^{2}$ & Follow-up comparisons \\
\hline $\begin{array}{l}\text { Receptive } \\
\text { language }\end{array}$ & TROG-E $^{\mathrm{a}}$ & $91.73(13.91)$ & $85.28(18.11)$ & $101.81(9.6)$ & $F_{(2,68)}=5.64, p<.01$ & .15 & $(\mathrm{SLI}=$ Apoor $)<$ Aapp \\
\hline $\begin{array}{l}\text { Expressive } \\
\text { language }\end{array}$ & ERRNI (MLUw) ${ }^{\mathrm{a}}$ & $97.21(17.78)$ & $86.33(14.96)$ & $93.75(10.62)$ & $F_{(2,68)}=2.84, p=.07$ & .08 & No further differences \\
\hline Oromotor & Oromotor sequences ${ }^{b}$ & $8.12(1.92)$ & $11.17(2)$ & $9.19(1.8)$ & $F_{(2,68)}=14.91, p<.01$ & .31 & $(\mathrm{SLI}=\mathrm{Aapp})<$ Apoor \\
\hline $\begin{array}{l}\text { Phonological } \\
\text { STM }\end{array}$ & Nonword repetition $^{\mathrm{b}}$ & $8.09(3.09)$ & $9.55(2.33)$ & $11.19(2.51)$ & $F_{(2.68)}=6.97, p<.01$ & .18 & $\begin{array}{l}\text { SLI < Aapp, no further } \\
\text { differences }\end{array}$ \\
\hline \multirow[t]{2}{*}{ CCC-2 } & GCC & $59.71(31.84)$ & $33.71(11.5)$ & $33.69(16.87)$ & $F_{(2,61)}=8.43, p<.01$ & .22 & $($ Aapp $=$ Apoor $)<\mathrm{SLI}$ \\
\hline & SIDC & $7.56(7.65)$ & $-8.29(9.58)$ & $-7.54(8.72)$ & $F_{(2,61)}=27.21, p<.001$ & .47 & $($ Aapp $=$ Apoor $)<$ SLI \\
\hline
\end{tabular}

${ }^{\mathrm{a}}$ Data are standard scores, based around a mean of 100 and a S.D. of 15.

b Data are standard scores, based around a mean of 10 and a S.D. of 3. 


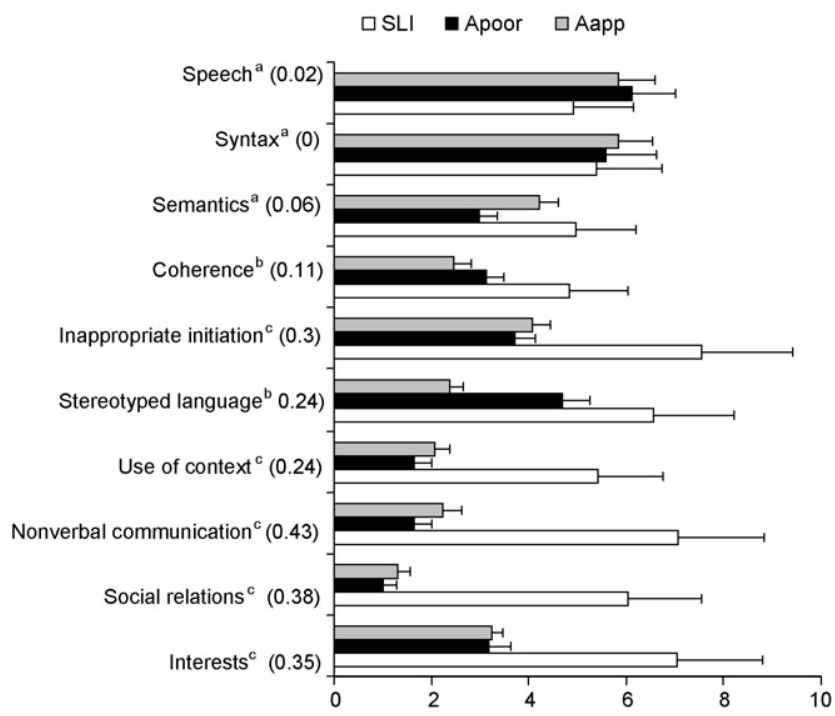

Fig. 1. Mean scaled scores of the SLI, Apoor and Aapp groups on the CCC-2 (based around a mean of 10 and a S.D. of 3). Error bars represent one SEM. Effect Sizes (partial eta ${ }^{2}$ ) are within parentheses. ${ }^{a}$, No differences; ${ }^{b}$, Aapp $<$ SLI; ${ }^{c}$, (Aapp = Apoor $)<$ SLI.

Separate one-way ANOVAs were used to investigate differences in the GCC and SIDC across the three clinical groups. Table 2 reports the main effects for group that emerged from these analyses. Scheffé post hoc tests found that the Aapp and Apoor groups had lower GCC scores than the SLI group (for both comparisons, $p<.001$ ), but did not significantly differ from each other $(p=.97)$. There were also significant differences in the SIDC scores of the three groups. The mean score of both autism subgroups was strongly negative, indicating the group was characterized by greater pragmatic than structural language difficulties. In comparison, the SIDC score of the SLI group was positive, indicating the presence of predominantly structural language difficulties.

\subsection{Errors on nonword repetition}

We then sought to compare the nonword repetition errors made by the participants in the SLI and Apoor groups who performed poorly on the test of nonword repetition. A standard score criterion of 8 or below was adopted to indicate poor performance on this task. There was no significant difference in chronological age, NVIQ or TROG-E performance between the 18 participants with SLI (chronological age: $M=12 ; 1$ S.D. $=2 ; 1$; NVIQ: $M=97.39$, S.D. $=11.64$; TROG-E: $M=90.33 ;$ S.D. $=14.52)$ and seven participants with autism (chronological age: $M=10 ; 4$, S.D. $=2 ; 7$; NVIQ: $M=90.33$; S.D. $=14.52$; TROG$\mathrm{E}: M=89 ;$ S.D. $=22.04$ ) who met this criterion (for all comparisons, $p>.75$ ).

The number of errors made by each participant was averaged across the various trials at each syllable length (i.e., three trials each for two-, three- and five-syllable nonwords, and four trials for four-syllable nonwords). The mean number of errors produced at each 


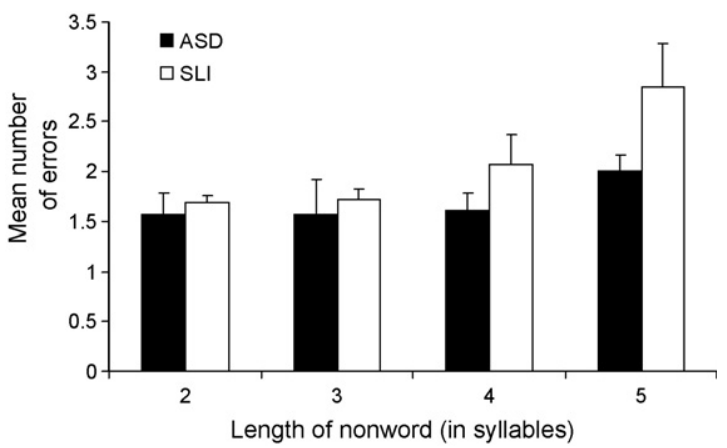

Fig. 2. Mean number of errors made by the children in the SLI and Apoor groups who had poor nonword repetition (standard score $\leq 8$; SLI: $n=18$; Apoor: $n=7$ ). Error bars represent one SEM.

syllable length is shown in Fig. 2. A repeated measures ANOVA revealed a main effect of syllable-length, $F(3,69)=13.59, p<.001$, while the main effect for group approached significance only, $F(1,23)=3.14, p=.09$. The interaction between syllable length and group was statistically significant, $F(3,69)=2.87, p<.05$, and demonstrated a clear linear trend, $F(1,23)=5.09, p<.05$.

We explored the linear trend by examining between-group differences at each syllable length. The two groups produced a similar number of errors for nonwords of two- and three-syllables (for both comparisons, $p>.5$ ). There was a statistical trend for the children with SLI to make more errors than the children with autism on the nonwords of foursyllables $(p=.12)$. This difference achieved statistical significance for the nonwords of five-syllables, $t(23)=2.29, p<.05$.

Hypothesis 2. Speech-motor difficulties in children with autism?

This second hypothesis predicts that poor nonword repetition in children with autism will be associated with speech motor deficits. To examine this proposal, we created four new groups based upon participants' diagnosis and their performance on the nonword repetition task. Once again, a standard score criterion of 8 or below was taken to indicate poor nonword repetition ability. The first and second groups comprised children with autism or SLI, respectively, who had appropriate performance on the nonword repetition task (autism: $n=25$; SLI: $n=16$ ). The third and fourth groups comprised children with each of these diagnoses who demonstrated poor nonword repetition (autism: $n=9$; SLI: $n=18)$. The four groups were statistically similar for chronological age, $F(3,64)=1.01$, $p=.39$, and nonverbal IQ, $F(3,64)=2.41, p=.08$ (see Table 3 ).

Hypothesis 2 will be supported if the children with autism who have poor nonword repetition show (1) similar results on the oromotor task to the two SLI subgroups, and (2) worse performance compared to the children with autism who do not demonstrate nonword repetition deficits. The performance of the four groups on this task is presented in Table 3 . A univariate ANOVA revealed a significant main effect of group, $F(3,64)=7.01, p<.001$. Both autism subgroups performed significantly better than the SLI subgroup with poor nonword repetition (for both comparisons, $p<.05$ ). A $t$-test comparing the performance of 
Table 3

Details of the ASD and SLI participants performing appropriately and poorly on the task of nonword repetition

\begin{tabular}{llllll}
\hline & \multicolumn{2}{l}{ Appropriate nonword repetition } & & \multicolumn{2}{l}{ Poor nonword repetition } \\
\cline { 2 - 3 } \cline { 5 - 6 } & ASD $(n=25)$ & SLI $(n=16)$ & & ASD $(n=9)$ & SLI $(n=18)$ \\
\hline Sex & 24 male, 1 female & 9 male, 7 female & & All male & 15 male, 3 female \\
Chronological age & $10 ; 9(2 ; 1)$ & $11 ; 8(2 ; 5)$ & & $10 ; 11(2 ; 8)$ & $12 ; 0(2 ; 1)$ \\
Range & $7 ; 3-15 ; 10$ & $7 ; 10-15 ; 8$ & & $7 ; 6-14 ; 3$ & $6 ; 11-14 ; 5$ \\
Nonverbal IQ & $107.83(14.93)$ & $103.69(14.42)$ & & $98.22(9.76)$ & $97.39(11.64)$ \\
Range & $80-137$ & $80-115$ & & $80-110$ & $82-137$ \\
Oromotor sequences $^{\mathrm{a}}$ & $10.24(2.24)$ & $8.39(2.15)$ & & $10.22(1.92)$ & $7.61(1.58)$ \\
\hline
\end{tabular}

For chronological age and nonverbal IQ, means and ranges are presented with standard deviations in parentheses. Mean (and S.D.) performance of the four groups on the oromotor sequences task is also presented here.

${ }^{a}$ Data are standard scores, based around a mean of 10 and a S.D. of 3.

the two autism subgroups on the oromotor sequences task found no significant difference, $t(32)=.02, p=.98$, Cohen's $d=0.01$.

Hypothesis 3. Substantial deficits in multiple autistic domains?

The third hypothesis predicts that, in the autistic population, there will be quantitative difference in autistic symptomatology between those children with and without nonword repetition deficits. If there is a difference between these two groups on the SCQ, which measures autism symptomatology, then this hypothesis would be supported. The autism subgroups were the same as those used in the investigation of Hypothesis 2 (see Table 3). Data on the SCQ were missing for five children in the autism subgroup with appropriate nonword repetition. However MANOVA showed that the chronological age, nonverbal IQ and performance on key behavioral measures (TROG-E, MLUw, repetition of nonwords) of these participants, were similar to that of the remaining participants in this group, $F(5,17)=1.78, p=.17$, Wilks' $\lambda=.66$.

An independent samples $t$-test revealed a between-group difference in SCQ scores, $t(26.57)=2.16, p<.05$. Scores on the SCQ indicated that the children with poor nonword repetition had significantly greater autistic symptomatology $(M=26.44$, S.D. $=3.84)$ than the children with appropriate nonword repetition $(M=21.8 ;$ S.D. $=7.74)$.

Whitehouse et al. (2007) proposed a synergistic relationship between the presence of considerable deficit in multiple autism domains and susceptibility to deficits in nonword repetition. To examine this idea, we investigated participants' scores on the three SCQ subscales (each subscale assessed one of the three autistic domains). First, we determined how many participants showed substantial impairment on each subscale. For this, we set a criterion of scoring a 'yes' on $50 \%$ or more of the questions relating to each subscale (i.e., social interaction subscale: scoring $\geq 8$ out of 15 ; communication subscale: scoring $\geq 7$ out of 13; restricted interests subscale: scoring $\geq 4$ out of 8 ). Participants in each group were then separated into two categories: either they exceeded this criterion on zero or 1 subscale or they exceeded it on two or three of the subscales.

Table 4 shows the number of children in the two autism subgroups who fell into each of these categories. All of the children in the poor nonword repetition group scored on $50 \%$ or 
Table 4

The number of children with autism who had either appropriate (NWRep+) or poor nonword repetition (NWRep-) and exceeded criteria on either zero/one subscale or two/three subscales of the SCQ

\begin{tabular}{|c|c|c|c|c|c|c|}
\hline & \multicolumn{3}{|l|}{$\geq 50 \%$} & \multicolumn{3}{|l|}{$\geq 67 \%$} \\
\hline & 0 or 1 subscale & 2 or 3 subscales & Total & 0 or 1 subscale & 2 or 3 subscales & Total \\
\hline NWRep+ & $11(55 \%)$ & $9(45 \%)$ & $20(100 \%)$ & $13(65 \%)$ & $7(35 \%)$ & $20(100 \%)$ \\
\hline NWRep- & $0(-)$ & $9(100 \%)$ & $9(100 \%)$ & $2(22.2 \%)$ & $7(77.8 \%)$ & $9(100 \%)$ \\
\hline
\end{tabular}

Proportions for each autism subgroup are shown within parentheses.

Note: $\geq 50 \%$ : scoring on $50 \%$ or more of the questions relating to a subscale; $\geq 67 \%$ : scoring on two-thirds or more of the questions relating to a subscale.

more on two or more subscales of the SCQ, as compared with only $45 \%$ of the group with appropriate nonword repetition. Fisher's exact test revealed these differences to be statistically significant $(p<.01)$. This between-group difference remained significant $(p<.05)$ even after we raised the criterion to require a positive score on two-thirds or more of the questions relating to each subscale (i.e., social interaction subscale: $\geq 10$ out of 15 ; communication subscale: scoring $\geq 9$ out of 13 ; restricted interests subscale: scoring $\geq 6$ out of 8). Seven participants with poor nonword repetition scored on two-thirds or more items on two or more subscales of the SCQ. All of these participants were classified as having language impairment.

\section{Discussion}

Poor nonword repetition is considered a psycholinguistic marker of SLI (Bishop et al., 1996; Conti-Ramsden et al., 2001). The finding that some children with autism also have deficits in nonword repetition (Kjelgaard \& Tager-Flusberg, 2001) has stimulated interest in whether there is a subgroup of children with autism who show a neurocognitive phenotype that is similar to children with SLI. The current study investigated this position (Hypothesis 1) and two alternative possibilities for nonword repetition difficulties in autism, i.e., that poor performance is associated with speech-motor difficulties (Hypothesis 2) or with greater severity of autistic symptomatology (Hypothesis 3).

Before the findings are discussed, it is important to discuss the limitations of the current study. First, because there was some (but not complete) overlap in the measures used to select the participants in the SLI and Apoor groups and the measures used as dependent variables, it could be considered unsurprising the these children perform poorly on the language tasks analyzed here. Importantly, however, the participants in these groups were selected based upon their poor performance on some (but not necessarily all) of a large range of standardized tests; they did not necessarily perform poorly on all language tasks. The current study was concerned with understanding the language profile of children with SLI (i.e., weaknesses and strengths across the various dependent measures) and how this compares with the language profile of the Apoor group. Second, because the participants were divided into different subgroups, the resulting sample sizes were small. The small sample sizes gave the study low power and raised the likelihood of making a type II error 
(incorrectly failing to reject the null hypothesis). This is especially relevant for the comparison of the SLI and Apoor groups on the task of nonword repetition; despite an apparent discrepancy in the standard scores of the two groups (SLI: $M=8.09$; S.D. = 3.09; Apoor: $M=9.55$; S.D. $=2.33$ ), the difference was not statistically significant. Similarly, the post hoc tests between SLI and the Apoor groups on the coherence subscale of the CCC-2, fell just short of significance (for both comparisons, $p=.08$ ). It is possible that with increased power (i.e., more participants in each group), these differences would achieve statistical significance. Third, there was a broad age-range of participants in each group. The pattern of language impairment in both autism and SLI can change with age (Bishop, 1994; Jarrold, Boucher, \& Russell, 1997), and the large developmental span of the participants in the current study may have influenced the findings. However, because the participants in the various subgroups had similar age-ranges, this could not explain the group differences. Finally, by selecting participants with autism for the current study only if they had a nonverbal IQ within normal limits, we may have underestimated language impairment in this population (i.e., language impairment tends to co-occur with poor nonverbal ability in children with autism; Jarrold et al., 1997). Note, however, that it was necessary to adopt this criterion in order to guard against the possibility that any language deficits observed in the children with autism were due to a more general cognitive impairment.

If there is an SLI subtype among children with autism (as claimed by Kjelgaard \& Tager-Flusberg, 2001), then it was expected that the SLI and Apoor groups would show similar linguistic strengths and weaknesses on the battery of language assessments (Hypothesis 1). In line with this hypothesis, the two groups demonstrated similar performance on the TROG-E as well as the MLUw and the structural language subscales of the CCC-2. These results replicate those of Kjelgaard and Tager-Flusberg (2001), who found that language-impaired children with autism have deficits in broad measures of receptive and expressive language ability. However, further analysis of the language profiles revealed a number of important differences between the two groups. In particular, the SLI group was found to have significantly worse performance than the Apoor group on the tasks of speech motor skill (oromotor sequences) and verbal STM (sentence repetition). Poor performance on these tasks is widely established in the SLI population and some researchers have postulated that these may also act as psycholinguistic markers of SLI (Barry et al., 2007; Conti-Ramsden et al., 2001). The finding that the Apoor group performed within normal limits on both of these tasks provides evidence against a shared phenotype between SLI and autism.

We then selected those participants in the SLI and Apoor groups who had poor nonword repetition. Examination of the nonword repetition errors made by these participants indicated further difference between groups. Although the two groups made a similar number of repetition errors for nonwords of two- and three- syllables, the SLI participants group tended to produce more errors than the autism participants for the longer nonwords. Central to the argument of Kjelgaard and Tager-Flusberg (2001) was the assumption that nonword repetition deficits in autism and SLI were due to the same cognitive limitation (i.e., phonological STM), and that this deficit was at least partly responsible for the similar language profiles of the two groups. However, the finding of a more gradual performance decline in the Apoor group indicates that there may be 
differences in the underlying mechanism(s) responsible for poor nonword repetition (and language impairment) in the autism and SLI populations. We suggest that these findings provide evidence against the proposal that there is a subtype among children with autism who have a language phenotype that is the same as that of children with SLI (TagerFlusberg \& Joseph, 2003).

The second hypothesis was that poor nonword repetition in autism was due to difficulty programming articulatory apparatus to execute a long and complex sequence of movements. Data from the current study do not support this position; the children with autism who had nonword repetition deficits performed equally well as the children with autism who had appropriate nonword repetition skills on the task of speech motor planning (oromotor sequences).

The third hypothesis for the origin of nonword repetition deficits in autism comes from the model of the relation between autism and SLI advanced in Whitehouse et al. (2007). Here it was argued that linguistic deficits in autism - including poor nonword repetition arise when an individual has significant impairment in more than one of the three autistic domains. The findings of the current study are consistent with this position; relative to the children with autism who had appropriate nonword repetition, the children with nonword repetition deficits were more likely to have significant deficit in multiple autistic domains. Further analysis found that the children with autism who had both poor nonword repetition and substantial deficits in two or more autistic domains, also had clinically significant impairments in structural language. However, while these findings indicate that increased autistic symptomatology tends to co-occur with both poor nonword repetition and language impairment, the data do not speak to a causal relationship. Research that investigates the association between autistic symptomatology and language ability over time will provide clarity to these ideas.

The results of this study require further exploration by future research. The framework outlined in Whitehouse et al. (2007) was proposed as one possibility for the origin of nonword repetition deficits in autism. Although the findings of this small-scale study provide preliminary support for this model, investigations by independent laboratories utilizing large sample sizes are essential for any clarity on these ideas. In particular, research should also seek to establish whether impairment in structural language (and poor nonword repetition) is associated with qualitative differences in autistic symptomatology. For example, are there specific combinations of autistic symptomatology that are particularly detrimental to language development? These ideas require testing on a range of children with autism, using a variety of measures assessing autistic features. For older children or adults with autism, assessments that provide a measure of autistic symptomatology during childhood (i.e., where the bulk of learning language occurs), such as the Autism Diagnostic Interview-Revised (Lord et al., 1994), would be particularly informative.

In summary, the findings of the current study provide evidence against the idea that there is an SLI subtype among children with autism. Instead the findings suggest that poor nonword repetition in autism is related to the presence of substantial deficit in multiple autistic domains. While there is a moderate risk of nonword repetition deficits with substantial deficit in a single autistic domain, this risk increases considerably with the presence of substantial deficit in two or more domains. 


\section{Acknowledgments}

The research was supported by grant from National Alliance for Autism ResearchAutism Speaks. Thank you to Tim Jones, Helen Flanagan, Tracy O'Donnell, David McDonald and Liz Line for their help during the testing phase of this project. Sincerest thanks to Emma Jaquet for her invaluable comments on draft versions of this manuscript. A special thanks to all children and their parents who selflessly gave up their time to participate.

\section{Appendix A. Question for continuing education}

1. Kjelgaard and Tager-Flusberg (2001) found nonword repetition deficits were apparent in:
a. all children with autism.
b. all children with autism except those who were echolalic.
c. children with autism who had a significant or borderline language impairment.
d. a handful of children with autism who had poor attention.
e. no child with autism.

2. So far, the molecular genetic evidence for overlap between SLI and autism is:
a. sentence repetition.
b. TROG.
c. oromotor ability.
d. MLU.
e. A and C.

a. scant: no evidence has been found for genetic overlap.
b. mixed: one study has found evidence for genetic association between the two disorders, but this has failed to be replicated by subsequent studies.

c. conclusive: it is clear that the two disorders share the same genetic liability.

3. On which tasks did the children with SLI and the children with autism who had structural language impairment differ?

4. We compared the nonword repetition errors made by the children with SLI and the children with autism who had both structural language impairment and poor nonword repetition. What did we find?
a. the children with autism made very few errors.
b. the children with autism made more errors than the children with SLI at each syllable length.
c. the two groups made a similar amount of errors for the shorter nonwords, but the children with SLI produced more errors for the nonwords of four- and five-syllables.
d. there was no difference between the groups.
e. none of the above.

5. According to the findings of this study, poor nonword repetition in children with autism is associated with:
a. poor attention.
b. the existence of an 'SLI subtype'. 

c. speech-motor difficulties.
d. substantial deficit in multiple autistic domains.
e. hearing impairments.

\section{References}

Alarcón, M., Cantor, R. M., Liu, J., Gilliam, T. C., the Autism Genetic Resource Exchange Consortium, \& Geschwind, D. H. (2002). Evidence for a language quantitative trait locus on chromsome 7q in multiplex autism families. American Journal of Human Genetics, 70, 60-71.

American Psychiatric Association. (1994). Diagnostic and statistical manual of mental disorders (4th ed.). Washington, DC: American Psychiatric Association.

Barry, J. G., Yasin, I., \& Bishop, D. V. M. (2007). Heritable risk factors associated with language impairments. Genes, Brain and Behavior, 6, 66-76.

Bishop, D. V. M. (1994). Is specific language impairment a valid diagnostic category? Genetic and psycholinguistic evidence. Philosophical Transactions of the Royal Society, series B, 346, 105-111.

Bishop, D. V. M. (1997). Uncommon understanding: development and disorders of language comprehension in children. Hove: Psychology Press.

Bishop, D. V. M. (2003). Children's communication checklist-2. London: Harcourt Assessment.

Bishop, D. V. M. (2004). Expression, reception and recall of narrative instrument. London: Harcourt Assessment.

Bishop, D. V. M. (2005). Test for reception of grammar: Electronic. London: Harcourt Assessment.

Bishop, D. V. M., Maybery, M., Wong, D., Maley, A., Hill, W., \& Hallmayer, J. (2004). Are phonological processing deficits part of the broad autism phenotype? American Journal of Medical Genetics: (Neuropsychiatric Genetics), 128B, 54-60.

Bishop, D. V. M., North, T., \& Donlan, C. (1996). Nonword repetition as a behavioural marker for inherited language impairment: Evidence from a twin study. Journal of Child Psychology and Psychiatry, 37, 391-403.

Cohen, J. (Ed.). (1988). Statistical power analysis for the behavioral sciences. Hilsdale, New York: Erlbaum.

Constantino, J. N., \& Todd, R. D. (2003). Autistic traits in the general population: a twin study. Archives of General Psychiatry, 60, 524-530.

Conti-Ramsden, G., Botting, N., \& Faragher, B. (2001). Psycholinguistic markers for specific language impairment (SLI). Journal of Child Psychology and Psychiatry, 42, 741-748.

Fein, D., Steven, M., Dunn, M., Allen, D., Rapin, I., Waterhouse, L., \& Feinstein, C. (1999). Subtypes of pervasive developmental disorder: clinical characteristics. Child Neuropsychology, 5, 1-23.

Folstein, S. E., Santangelo, S. L., Gilman, S. E., Piven, J., Landa, R., Lainhart, J., et al. (1999). Predictors of cognitive test patterns in autism families. Journal of Child Psychology and Psychiatry, 40, 1117-1128.

Gathercole, S. E., \& Baddeley, A. D. (1990). Phonological memory deficits in language disordered children: Is there a causal connection? Journal of Memory and Language, 29, 336-360.

Graf-Estes, K., Evans, J. L., \& Else-Quest, N. M. (2007). Differences in the nonword repetition performance of children with and without specific language impairment: A meta-analysis. Journal of Speech Language and Hearing Research, 50, 177-195.

Jarrold, C., Boucher, J., \& Russell, J. (1997). Language profiles in children with autism: theoretical and methodological implications. Autism, 1, 57-76.

Kjelgaard, M. M., \& Tager-Flusberg, H. (2001). An investigation of language impairment in autism: Implications for genetic subgroups. Language and Cognitive Processes, 16, 287-308.

Korkman, M., Kirk, U., \& Kemp, S. I. (1998). NEPSY: A developmental neuropsychological assessment. San Antonio: Psychological Corporation.

Lai, C. S. L., Fisher, S. E., Hurst, J. A., Vargha-Khadem, F., \& Monaco, A. (2001). A forkhead-domain gene is mutated in a severe speech and language disorder. Nature, 413, 519-523.

Lord, C., Risi, S., Lambrecht, L., Cook, E. H., Leventhal, B. L., DiLavore, P. C., et al. (2000). The autism diagnostic observation schedule-generic: A standard measure of social and communication deficits associated with the spectrum of autism. Journal of Autism and Developmental Disorders, 30, 205-223. 
Lord, C., Rutter, M., \& Le Couteur, A. (1994). Autism diagnostic interview-revised: A revised version of a diagnostic interview for caregivers of individuals with possible pervasive developmental disorders. Journal of Autism and Developmental Disorders, 24, 659-685.

Newbury, D. F., Bonora, E., Lamb, J. A., Fisher, S. E., Lai, C. S. L., Baird, G., et al. (2002). FOXP2 is not a major susceptibility gene for autism or specific language impairment. American Journal of Human Genetics, 70, 1318-1327.

Page, J., \& Boucher, J. (1998). Motor impairments in children with autistic disorder. Child Language Teaching and Therapy, 14, 233-259.

Pilowsky, T., Yirmiya, N., Shalev, R. S., \& Gross-Tsur, V. (2003). Language abilities of siblings of children with autism. Journal of Child Psychology and Psychiatry, 44, 914-925.

Ronald, A., Happé, F., Bolton, P., Butcher, L. M., Price, T. S., Wheelwright, S., Baron-Cohen, S., \& Plomin, R. (2006). Genetic heterogeneity between the three components of the autism spectrum: A twin study. Journal of the American Academy of Child and Adolescent Psychiatry, 45, 691-699.

Rutter, M., Bailey, A., \& Lord, C. (2003). Social communication questionnaire (SCQ). Los Angeles: Western Psychological Services.

Semel, E., Wiig, E. H., \& Secord, W. H. (1995). Clinical evaluation of language fundamentals (3rd ed. (CELF-3)). San Antonio, TX: Psychological Corporation.

Tager-Flusberg, H. (2006). Defining language phenotypes in autism. Clinical Neuroscience Research, 6, $219-224$.

Tager-Flusberg, H., \& Cooper, J. (1999). Present and future possibilities for defining a phenotype for specific language impairment. Journal of Speech, Language and Hearing Research, 42, 1001-1004.

Tager-Flusberg, H., \& Joseph, R. (2003). Identifying neurocognitive phenotypes in autism. Philosophical Transactions of the Royal Society of London: Series B, 358, 303-314.

Tomblin, J. B., Hafeman, L. L., \& O’Brien, M. (2003). Autism and autism risk in siblings of children with specific language impairment. International Journal of Language and Communication Disorders, 38, 235-250.

Torgesen, J. K., Wagner, R., \& Rashotte, C. (1999). Test of Word Reading Efficiency (TOWRE). New York: Psychological Corporation.

Wassink, T. H., Piven, J., Vieland, V. J., Pietela, K., Goedken, R. J., Folstein, S. E., et al. (2002). Evaluation of FOXP2 as an autism susceptibility gene. American Journal of Medical Genetics (Neuropsychiatric Genetics), 114, 566-569.

Wechsler, D., \& Chen, H.-Y. (1999). Wechsler abbreviated scale of intelligence. New York: The Psychological Corporation.

Whitehouse, A. J. O., Barry, J. G., \& Bishop, D. V. M. (2007). The broader language phenotype of autism: A comparison with specific language impairment. Journal of Child Psychology and Psychiatry, 48, 822-830. 